\title{
Ueber die specifische Lichtabsorption des gelben Fleckes der Netzhaut.
}

\author{
Von \\ Dr. Moriz Sachs, \\ Assistent am deutschen physiologischen Institut in Prag. \\ Hierzu Tafel VI. \\ I.
}

Vor der sehtüchtigsten Stelle der menschlichen Retina ist das Pigment der Macula lutea gelagert; alle Strahlen, welche zu den lichtempfindlichen Gebilden dieses Retinabezirkes gelangen sollen, müssen vorher das Pigment der Macula passirt haben, wobei die verschiedenen Strahlenarten eine verschieden starke Absorption erleiden. Von dieser electiven Absorption kann man sich am leichtesten tiberzeugen, wenn man eine möglichst frische Macula sammt angrenzender ungefärbter Retina auf einem Objectträger ausbreitet und das Präparat bei verschieden farbigem Lichte und schwacher Vergrösserung untersucht: dies entweder nach $\mathrm{H}$ e $\mathrm{r}$ i $\mathrm{n} \mathrm{g}$ mit Hülfe von Vorrichtungen zur microscopischen Untersuchung im homogenen Lichte, oder in Ermangelung homogenen Lichtes nach dem Vorgange Max Schultze's durch Einschaltung gefärbter Gläser zwischen Spiegel und Objecttisch des Microscops. Lässt man das Präparat von gelbem Lichte durehstrahlen, so gelingt es nicht, die Gegend der Macula von der umgebenden Retina zu unterscheiden; anders, wenn man Strahlen kiirzerer Wellenlänge durchs Präparat schickt, die von der Macula latea theilweise absorbirt werden und diese viel dunkler als die umgebende pigmentfreie Netzhaut erscheinen lassen.

Diese Absorption von Seiten des Maculapigmentes, die auch im lebenden Auge stattfindet (der gelbe Fleck ist keine Leichenerscheinung) hat zur Folge, dass sich die dahinter gelegene Retina 
Ueber die specifische Lichtabsorption des gelben Fleekes der Netzhant. 575

bei Belichtung mit einer Strahlenart, die vom Pigment theilweise absorbirt wird, für diese Strablenart als relativ unterempfindlich gegeniiber der umgebenden Retina erweist. Die farbige Absorption der Macula ist nämlich nicht wettgemacht durch eine entsprechend abweichende Entwickelung des Farbensinns des macularen Netzhautgebietes, derart, dass der Empfindungswerth für eine bestimmte Intensität einer Strahlenart trotz ihrer theilweisen Absorption der gleiche wäre für die maculare wie für die extramaculare Retina.

Es geht dies aus einer Reihe von Thatsachen hervor, die alle beweisen, dass die Umgebung der Macula im Vergleich zu dieser empfindlicher ist für kurzwellige Strahlen.

Mischt man sich z. B. auf dem Farbenkreisel für das directe Sehen ein reines Griin aus einem hierzu geeigneten Blangrïn und Gelbgrün, so erscheint jenes Grün bläulich gefärbt, wenn man es mit einer extramacularen Parthie der Netzhaut betrachtet.

Noch übersichtlicher liegen die Verhältnisse in Fällen, wo die Macula lutea als subjective Gesichtserscheinung anftritt. Bekanntlich erscheint, wenn man durch ein blaues Glas sieht, das Centrum des Gesichtsfeldes als dunkler Fleck; die Grösse des letzteren entspricht ungefähr der Ausdehnung der Macula lutea; manchmal scheint der Fleck geradezu gelb zu sein; die Gelbempfindung tritt dann infolge des Contrastes zwischen intensiverem and minder intensivem Blau auf.

$\mathrm{Da}$, wie ans dem Angeführten hervorgeht, die farbige $\mathrm{Ab}$ sorption durch die Macula nicht compensirt wird durch eine eigenthümliche Entwickelung des Farbensinns an der dahinter gelegenen Retinaparthie, so muss eine Verschiedenheit der Menge des Maculapigmentes auch eine Verschiedenheit der Reizwerthe der in Betracht kommenden Strablungen zur Folge haben, das heisst aber nichts anderes, als dass mit dem Nachweiss von individuellen Verschiedenheiten in der Färbung der Macula auch der Beweis für das Bestehen individueller Verschiedenheiten des Farbensinns. erbracht ist. Max Schultze ${ }^{1}$ ) sprach unter Hinweiss auf das wechselnde Verhalten der Pigmente im thierischen und menschlichen Körper die Vermuthung aus, dass wahrscheinlich auch das Maculapigment in seiner Intensität und Extensität individuellen

1) Ueber den gelben Fleck der Retina. Bonn 1866. 
Schwankungen unterliege. Er war sich darüber klar, dass solche Schwankungen auf die Farbenempfindung von Einfluss sein miissen, doch fehlte es ihm an physiologischen Thatsachen, die er mit der von ihm vermutheten Inconstanz der Entwickelung des Maculapigmentes hätte in Zusammenhang bringen können. Er begnügte sich damit, die mehrfach beobachtete Violettblindheit, sowie die veränderte Farbenempfindung im Santoninrausch durch eine stärkere Färbung der Macula zu erklären. Den umgekehrten Weg nahmen die Untersuchungen späterer Forscher; sie führten von der Erkenntniss der individuellen Verschiedenheiten des Farbensinns zur Erklärung der letzteren aus dem wechselnden Verhalten des Pigmentes der Vacula und der Linse.

Individuelle Verschiedenheiten des Farbensinns wurden wohl 'mehrfach beobachtet, zumal bei Rothgrümblinden, jedoch zum geringsten Theil als solche aufgefasst. Maxwell ${ }^{1}$ ) fand als erster, dass bei Herstellung gewisser Gleichungen zwischen je drei Spectralfarben und weissem Licht bei verschiedenen farbentiichtigen Beobachtern verschiedene Mengenverhältnisse der verwendeten spectralen Farben erforderlich waren. Er suchte dieses abweichende Verhalten aus einer individuell verschieden starken $\mathrm{Ab}$ sorption durch die Macula zu erklären, ohne diese Annahme durch weitere Grïnde zu stïtzen.

Nach ibm fand diese Frage durch v. Frey und v. Kries $\left.{ }^{2}\right)$ eine neuerliche Bearbeitung; die genannten Autoren fanden, dass zwischen ihnen gesetzmässige Untersehiede der Farbenempfindung bestehen, die sich aus der einfachen Annahme erklären .. lassen, dass „zu den percipirenden Elementen des einen von ihnen (K.). ein gelberes Licht als zu.denen des anderen gelange ${ }^{*}$. Die gefundenen Unterschiede der Farbenempfindung sollten ihrer Ansicht nach durch eine verschieden starke Absorption der blanen Strablen von Seiten der Macula bedingt sein. Einen Beweis für diese Annahme erblickten sie darin, dass Unterschiede der gleichen Art wie sie für die Centren ibrer Netzhäute nachzuweisen waren, auch zwischen Netzhautcentrum und Peripherie jedes einzelnen von ihnen bestehen, was soviel heisst, als dass die individuellen Unter-

1) Philosophical Transactions 1860.Bd. CL. 57-84.

2) Ueber die Mischung von Spectralfarben. du Bois-Reymond's Archiv f. Physiol. 1881. S. 336. 
scbiede in der Farbenemptindung nur im Maculabereich der Retina in Erscheinung treten.

In einer viel directeren Weise suchte $\mathrm{Hering}{ }^{1}$ ) den Zusammenhang zwischen den individuellen Verschiedenheiten des Farbensinns und der wechselnd starken Pigmentirung der Macula zu erweisen.

Seine diesbezüglichen Untersuchungen gingen von der grïndlichen Erforschung der Breite des Vorkommens und insbesondere der Grösse der individuellen Verschiedenheiten des Farbensinns aus.

Diese Verschiedenheiten änssern sich nach Hering vorwiegend darin, dass die blauwerthigen Strahlen für verschiedene Individuen eine verschieden grosse blaue Valenz besitzen; ein Grün, gemischt aus spectralem Grüngelb und spectralem Grünblau, das der Eine rein grün empfindet, kann einem Anderen blänlich-grün, einem Dritten gelblich-grün erscheinen; hierbei muss natürlich der Einfluss einer verschiedenen Stimmung der betreffenden Sehorgane möglichst ausgesehlossen sein.

Hering nannte nun von zwei Farbentüchtigen jenen, für den die blauwertbigen Strahlen einen relativ grossen Reizwerth besitzen im Vergleich zum andern relativ blausichtig; der andere ist dem blausichtigen gegenüber als relativ gelbsichtig zu bezeichnen.

In der relativen Blausichtigkeit und der relativen Gelbsichtigkeit gipfeln nur die individuellen Verschiedenheiten des Farbensinns. Eine genauere Untersuchung lehrte nämlich, dass sich die relative Ueberempfindlichkeit der Blansichtigen für Strahlen kürzerer Wellenlänge bereits im Gelbgriin geltend macht; der relativ blausichtige ist sonach stets auch relativ grünsichtig, der relativ gelbsichtige gleichzeitig relativ rothsichtig und die Bezeichnungen "blausichtig" und "gelbsichtig“ sind nur a fortiori zu nehmen. Nebenbei sei bemerkt, dass die Untersuchungen in ibrer Methode dem Einwande begegneten, dass die gefundenen Unterschiede bloss Folge einer individuell wechselnden Bezeichnung für im übrigen gleiche Farbenempfindungen wären.

Um nun die Abhängigkeit der gefundenen Unterschiede von der Färbung der Macula zu demonstriren, entnahm Hering menschlichen Augen, die kurz nach dem Tode exstirpirt waren, die Netz-

1) Ueber individuelle Verschiedenheiten des Farbensinnes. Lo tos Neue Folge Bd. VI. 1885. 
haut der Gegend der Macula Intea, breitete dieselbe auf einem Objectträger aus, liess sie vorsichtig antrocknen und schloss sie dann unter Glycerin ein. Es zeigte sich, dass die auf diesem Wege gewonnenen Maculae luteae nicht gleich intensiv gefärbt waren. Hering liess nun die relativ blausichtigen Versuchspersonen durch eine passend gewählte Macula beobachten; jetzt stimmten sie in ihren Angaben mit den relativ gelbsichtigen mehr oder weniger genau überein. Je grösser die Blausichtigkeit des Betreffenden war, eine desto intensiver. gefärbte Macula musste gewählt werden, um Gelbsichtigkeit zu produciren. Die genannten Verschiedenheiten der Farbenempfindungen zweier Individuen verschwanden also, wenn der Blausichtigere durch eine passend gewählte Macula beobachtete. Hieraus ergiebt sich, dass seine relative Blausichtigkeit lediglich die Folge einer schwächeren Pigmentirung seines gelben Fleckes sein kann.

Ebenso beweisend wie diese Versuche sind die eingangs kurz erwähnten Untersuchungen der Macula lutea im Microspectrum. Es lässt sich auf diesem Wege mit Bestimmtheit nachweisen, dass die Macula lutea nicht bloss die violetten und blauen, sondern auch die grtinen Strahlen absorbirt. Viel empfindlicher als die Untersuchung im Microspectrum ist eine andere von Hering angewendete Methode; sie beruht darauf, dass Gleichungen zwischen einem homogenen Lichte und einer Mischung aus zwei homogenen Lichtern zerstört werden, wenn bei der Betrachtung der Gleichung: durch eine vor's Auge gebaltene Macula, die drei verwendeten Lichter nicht im gleichen Verhältniss absorbirt werden. H e r i n $g$ konnte so den Nachweis führen, dass sich die Absorption durch das Maculapigment noch auf Licht von der Wellenlänge $565 \mu \mu$ erstreckt. Diese Methode lässt sich nur für Lichter vom rothen Ende bis etwa zum Grün von der Linie $\mathrm{E}$ anwenden, weil andernfalls das Mischlicht viel weniger gesättigt ist als die homogene Strahlung, und eine Ausgleichung dieser Sättigungsdifferenz durch Zusatz von Weiss zum homogenen Lichte sich von selbst verbietet.

Also ebenso wie sich die Ueberempfindlichkeit der relativ Blausichtigen für Strahlen kürzerer Wellenlänge noch im Gelbgrün constatiren lässt, lässt sich die Absorption von Seiten des Maculapigmentes bis in's.Gelbgriun verfolgen, und ganz entsprechend dem Befunde, dass die relative Grünsichtigkeit der Blausichtigen ihrer Blausichtigkeit gegenïber an Bedeutung sehr nachsteht, findet man, 
dass die Macula lutea die blauen Strahlen viel stärker absorbirt als die grünen und vollends die gelbgrünen.

Besondere Bedeutung erhielten die Untersuchnngen Hering's dadurch, dass sie den Einfluss der individuellen Verschiedenheiten des Farbensinns and damit der wechselnden Färbung der Macula auf das Zustandekommen des wechselnden Bildes der Rothgrïnblindheit darlegten.

Es gelang Hering, den Nachweis zu führen, dass der Scheidung der partiell Farbenblinden in Rothblinde und Grüinblinde eine irrige Auffassung zu Grunde lag. Jeder Defect des Rothsinns ist unbedingt verknïpft mit einem Defect des Grünsinns und umgekehrt, wie sich dies aus der Hering'schen Annahme eines einheitlichen Rothgränsinns von selbst ergibt.

Die angebliche Grünsichtigkeit der sogenannten Rothblinden läuft auf eine relative Blausichtigkeit, die scheinbare Rothsichtigkeit der Grünblinden auf eine relative Gelbsichtigkeit der betreffenden Individuen hinaus.

In ganz ähnlicher Weise; wie Hering einen blausichtigen Farbentüchtigen dadurch, dass er ihn durch eine passende Macula beobachten liess, relativ gelbsichtig machen konnte, konnte er auch einen sogenannten Rothblinden (blausichtigen Rothgrünblinden) in einen Grünblinden (gelbsichtigen Rothgruinblinden) verwandelu.

Es ist damit höchst wahrscheinlich gemacht, dass die bei Rothgrünblinden nachgewiesenen Unterschiede von der gleichen Art sind wie jene, welche Individuen mit normaler Farbenempfindung untereinander zeigen, gleichviel ob diese Unterschiede nur in verschiedener Pigmentirung der Macula bezw. der Linse oder noch anderweit begründet sind.

Obgleich die durch das Maass der Pigmentirung der Macula bedingten Verschiedenheiten des Farbensinns nicht die einzig vorkommenden sind, so sind sie doch für die Stelle des schärfsten Sehens von dominirender Bedeutung. Mit Rücksicht auf die hervorragende Dignität dieser Sehstelle erschien es von Interesse; die Grösse der specifischen Absorption der Macula und damit die Grösse der durch diese Absorption veranlassten individuellen Verschiedenheiten der Farbenempfindung festzustellen.

Der grossen Freundlichkeit meines hochverebrten Lehrers, des Herrn Professor $\mathrm{H}$ e r i n g verdanke ich die Ueberlassung dieses Themas, dessen Bearbeitung er bereits in seiner Schrift „Ueber 
die individuellen Verschiedenheiten des Farbensinnes ${ }^{u}$ in Aussicht gestellt hatte.

\section{II.}

Aus dem Vorhergehenden ist zur Genüge ersichtlich, dass sich die Frage nach der Grösse der Absorption der Macula lutea zum Theil deckt mit der Frage nach der Grösse der individuellen Verschiedenheiten des Farbensinns, derart, dass es principiell gleichgiltig ist, für welehe der beiden Fragen die Lösung gesucht wird.

Es muss daher erwogen werden, was zu thun räthlicher ist: die Grösse der individuellen Verschiedenheiten des Farbensinnes zu bestimmen oder die Grösse der specifischen Absorption der Macula festzustellen.

Man könnte daran denken, die Grösse der Verschiedenheiten in den Farbenempfindungen eines relativ Blausichtigen und eines Gelbsichtigen zu messen, oder den Einfluss der Tinction der Macula auf die Farbenempfindung dadurch in messender Weise zu bestimmen, dass man an ein und demselben Individuum die Farbenempfindungen des macularen und eines extramacularen Theiles der Netzhant vergleicht.

Die bier in Betracht kommenden Methoden, die sich zwar selur wohl zur Feststellung qualitativer Unterschiede in der Farbenempfindung eignen, lassen sich jedoch nur in einem kleinen Theile des Spectrums messend durchführen. Im folgenden soll dies noch näher begriindet werden, hier will ich nur kurz andeuten, dass der Grund dafür darin zu suchen ist, dass sich die individuellen Verschiedenheiten in einem zu grossen Theile des Spectrums geltend machen.

Will man die vorliegende Frage entscheiden, so bleibt demnach nichts anderes übrig, als die Grösse der Absorption durch die Macula direct zu bestimmen.

Im folgenden ist eine Darstellung des Ganges und der Resultate einer solchen Untersuchung gegeben; die ausserordentliche Zuvorkommenheit der Vorstände der pathologisch - anatomischen Institute zu Wien und zu Prag ermöglichte es mir, in kurzer Zeit in den Besitz einer grösseren Anzahl von Maculae lateae zu gelangen. Dieselben wurden meist drei Stunden nach dem Tode ausgeschnitten und in der oben angegebenen Weise conservirt. Be- 
reits mit blossem Auge erkennt man die grossen individuellen Verschiedenheiten in der Färbung der Maculae; anch hat es den Anschein, als ob auch die Ausdehnung der Pigmentirung grossen Schwankungen unterliegen würde. Trotz der Bearbeitung eines grossen Materials konnte ich niemals die Angabe Max Schultze's bestätigt finden, dass die Fovea der stärkst pigmentirte Theil der Netzhaut ist; vielmehr fand ich ganz ausnahmslos die Fovea überhaupt unpigmentirt. Dagegen ist die unmittelbare Umgebung der Fovea anscheinend am stärksten pigmentirt. Die gelben Flecke wurden im Dunkeln aufbewahrt und nur zur Untersuchung an's Licht gebracht. Die eigentliche Untersuchung wurde nach einer mir von Prof. Hering empfohlenen Methode an einem Spectralapparat mit zwei Collimatoren gemacht. Jeder dieser Collimatoren fïllt eine Hälfte des kleinen kreisförmigen Gesichtsfeldes mit homogenem Lichte, und zwar der rechte Collimator die linke Hälfte, der linke Collimator die rechte Hälfte desselben. Als Lichtquelle diente gespiegeltes Himmelslicht; das Licht wurde für beide Collimatoren von angenähert derselben Himmelsstelle genommen.

Die Untersuchung wurde nur bei gleichmässig blauem Himmel vorgenommen. Vor dem Spalte des rechten Collimatorrohres wurde der Objectträger mit der Macula in passender Weise befestigt, so dass alles Licht, das den Spalt erleuchtete, die Macula vorher passiren musste. Falsches Licht war dadureh fast ganz ausgeschlossen, dass der Objectträger bis auf die am intensivsten gefärbte Stelle der Macula mit schwarzem Papier beklebt war.

Nachdem die Wellenlängen für jedes der beiden Spectren durch Einstellung auf die Franenhofer'schen Linien mittels Spiegelablesung ermittelt worden waren, wurde das Ocular des Fernrohrs entfernt. Das Auge des Beobachtenden wurde dicht an einen schmalen rechteckigen Spalt gebracht, in dessen Ebene das Spectrum lag. Der Ocnlarspalt batte eine Höhe von $2 \mathrm{~mm}$ und eine Breite von ca. $1 / 3 \mathrm{~mm}$. Das kreisförmige Gesichtsfeld, dessen rechte und linke Hälfte durch eine verticale, der Prismenkante entsprechende Linie geschieden ist, hatte in eine Entfernung von $20 \mathrm{~cm}$ projicirt einen scheinbaren Durchmesser von 1,5 cm. Die Breite der rechten Collimatorspalte, vor welcher die Macula lag, blieb während der ganzen Untersuchung unverändert.

Beide Collimatoren wurden zunächst auf Licht eingestellt, dessen mittle Wellenlänge derjenigen der D-Linie gleich war. 
Durch Verstellung der Schraube des linken Collimatorspaltes wurde jene Spaltbreite ermittelt, bei welcher beide Gesichtsfeldhälften vollstündig." gleich "aussahen; um Zufälligkeiten auszuschliessen, wurden stets mehrere Einstellungen gemacht. Die Grösse der gefundenen Spaltbreiten konnte an der Mikrometerschraube abgelesen werden. Die für D ermittelte Spaltbreite des linken Collimator ist im folgenden allemal gleich 1 gesetzt. Wurden nun beide Kreisbälften mit demselben lichte kürzerer Wellenlänge gefiillt, so machte sich die Absorption dieses Lichtes von Seiten der Macula dadurch geltend, dass die linke Hälfte des kreisförmigen Gesichtsfeldes dunkler erschien als die rechte Hälfte. Die rechte Gesichtsfeldhälfte wurde jetzt der linken wieder gleich gemacht durch Verengerung der linken Collimatorspalte. Die Grösse der nothwendigen Verengerung konnte als Maass der Absorption des verwendeten Lichtes durch die Macula benïtzt werden.

Im ganzen wurden neun Maculae einer genaueren Untersuchung unterzogen. Da es aus mehreren Gründen - Wechsel des Himmels, Beleuchtung etc. - wünschenswerth erschien, die Untersuchung je einer Macula in einer Sitzung zu erledigen, so konnte sich dieselbe jedesmal nur auf eine begrenzte Anzahl von Lichtern erstr ecken.

Nachdem einmal der grobe Verlanf der Absorptionscurve ermittelt war, wurde das Hauptgewicht der Untersuchung auf jenen Spectraltheil gelegt, in dem sich die Grösse der Absorption am raschesten ändert. Die Absorption nimmt zu mit abnebmender Wellenlänge, jedoch nicht gleichmässig. Zwischen D und E steigt sie langsam an, nimmt dann zwischen $E$ und $F$ rasch zu, so dass sie in $\mathrm{F}$ bereits nahezu das Maximum erreicht hat und bleibt dann annähernd gleich gross bis in's Violett hinein.

Im Grünelb ist die Absorption bereits so deutich, dass sie sich messen lässt, in zwei Fällen wurde die Grösse der Absorption bestimmt; sie betrug für Licht von der Wellenlänge $560 \mu \mu 1 / 30$ und $1 / 20$ der verwendeten Lichtmenge - die Lichtmenge bei der Linie D gleich 1 gesetzt - für Licht von der Linie $\mathrm{E}$ beträgt die Absorption $1 / 15-1 / 9$; für $\mathrm{F} 1 / 5_{5}-2 / 5$; für $g$ ist sie etwas grösser.

Ganz regelmässig nahm die Absorption am raschesten zu zwischen $518,3\left(b_{1}\right)$ und $505 \mu \mu$. Die Untersuchung von Lichtern, die langwelliger sind als $\mathrm{D}$, nahm ieh nur einmal vor; die bei $\mathrm{D}$ ermittelten Spaltureiten brauchten nicht verändert zu werden; dies 
Ueber die specifische Lichtabsorption des gelben Fleckes der Netzhaut. 583

war im vorhinein zu erwarten, da Hering mit Hülfe einer sehr empfindlichen Methode keine Absorption der rothen Strahlen durch die Macula nachutweisen im Stande wal. Ich habe infolge dessen von einer jedesmaligen Prífung rother Lichter auf ihre Absorption Abstand genommen.

Trägt man als Abscissen die Wellenlängen, als Ordinaten die gefundenen Spaltbreiten ein, so erhält man ein auschauliches Bild vom Verlaufe der Absorption im Spectrum.

Ich gebe im folgenden einige solcher "Absorptionscurven" wieder. Taf. VI.

Alle Curven warden für D auf denselben Werth gebracht; trotzdem zeigen sie im Einzelnen nicht unbedeutende Abweichungen, welche stch nicht alle auf quantitative Verschiedenheiten des Pigmentes zurickfübren lassen. Ich will es dahingestellt sein lassen, ob dies aus qualitativen Verschiedenheiten der Absorption oder aus der Breite möglicher Versuchsfehler zu erklären ist.

Im Grossen und Ganzen haben aber die Curven denselben Verlauf; es zeigt sich, dass die Absorption von Seiten der Macula fiur einen grossen Theil des Spectrums in Betracht kommt, and dass

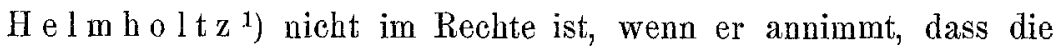
Absorption der Macula nur für Licht von der Linie $F$ von nennenswerther Bedentung ist. Der Umstand, dass die Absorption durch die Macula für einen grossen Theil des Spectrums in Betracht zu ziehen ist, hat, wie bereits oben angedentet wurde, zur Folge, dass sich die Grösse ber individuellen Versehiedenheiten zweier Augen im Leben nicht ohne weiteres messen lässt. Man kaun wohl eine Gleichung zwischen einem homogenen Gelb und einer Mischung von Roth oder Orange und Gelbgriun nacheinander von einem Blausichtigen und einem Gelbsichtigen einstellen lassen. Ist die Menge des Gelb und die des Orange gegeben, so werden die beiden Beobachter verschiedene Mengen von Gelbgrün brauchen, um eine Gleichung herzustellen. Die Gleichung enthält nur eine Unbekannte - in diesem Falle die zu suchende Menge des Gelbgrün. Der Unterschied in der Menge des für beide Beobachter erforderlichen Gelbgruin wäre ein Maass der verschieden grossen Valenz der gelbgrünen Strablung für diese beiden Individuen.

Wollte man aber in ähnlicher Weise auch fïr andere Lichter

1) Phys, Optik II. Aufl. pag. 382. 
die mit dem Individuum wechselnde Grösse der Valenz bestimmen, so würde dies daran scheitern, dass sich die individuellen Versehiedenheiten des Farbensinns anf beiden Seiten der Gleichung bemerkbar machen würden. Derartige Gleichungen enthalten bloss dann nur eine Unbekannte, wenn zwei von den drei zur Verwendung kommenden Lichtern langwelliger als Licht von ca. $560 \mu \mu$ sind. Durch diese Einschränkung wird aber die Zahl der mög. lichen Gleichungen eine ausserordentlich begrenzte und der für die Entscheidung der oben gestellten Frage wichtigste Theil des Spectrums kann gar nicht in Untersuchung gezogen werden. Von diesem Gesichtspunkte aus muss man nach Hering die Thatsache zu verstehen suchen, dass eine Gleichung zwischen Gelb und Blau einerseits und dem weissen Tageslicht anderseits sowohl für rel. Gelbsichtige als auch für rel. Blausichtige stimmen kann, indem die durch Absorption herbeigeführte Reduction des Reizwerthes für das homogene Blau ebenso gross ist wie im Durchschnitt für die Gesammtheit der blauwerthigen Strahlen des reinen Tageslichtes. Dazu kommt, dass die Gleichung überhaupt nicht sehr empfindlich ist; die Herstellung eines Mischweiss aus Gelb und "Blau ist auch fur ein und dasselbe Individuum innerhalb relativ grosser Grenzen des Mischungsverhältnisses möglich, weil die beiden hier in Betracht kommenden Lichter im Verhältniss zu ihrer farbigen Valenz grosse weisse Valenzen besitzen, welche sich bei der Mischung summiren, und durch ihr Uebergewicht etwa vorhandene Reste von farbiger Valenz vernichten.

Eine Messung der Grösse der Verschiedenbeiten des Farbensinns zwischen einem rel. Gelbsichtigen und einem rel. Blausichtigen wäre höchstens noch durchführbar, wenn die zu Untersuchenden mit adaptirtem Auge beobachten würden. In diesem Falle lassen sich Gleichungen zwischen je zwei beliebigen homogenen Lichtern herstellen, weil nur die weissen Valenzen der Lichter optisch verwertet- werden. Eine ähnliche, wenn auch nicht ad hoc angestellte Untersuchung rührt von $\mathrm{Her}$ in $\mathrm{g}^{1}$ ) her.

He ring ermittelte die Helligkeitskurve des Spectrums für einen total Farbenblinden und verglich sie mit der Curve der weissen Valenzen, die er mit seinem für Dunkel adaptirten Auge festzustellen suchte. Er fand, dass die Curven, die im Grossen

1) Untersuchung eines total Farbenblinden. Dieses Arch. Bd, 49. S. 563. 
Ueber die specifische Lichtabsorption des geiben Fleckes der Netzhaut. 585

und Ganzen einen identischen Verlauf zeigten, im blauwerthigen Theile des Spectrums gegeneinander etwas verschoben waren, was H e ring aus seiner relativen Gelbsichtigkeit zu erklären suchte. Die Unterschiede wären noch bedeutender gewesen, wenn nicht als Messlicht das Licht von der Linie $\mathrm{E}$ verwendet worden wäre, das im gelbsichtigen Auge bereits einer nicht unbedeutenden $\mathrm{Ab}$ sorption unterliegt.

Will man auf diesem Wege einen genaueren Aufschluss über die Grösse der individuellen Verschiedenheiten erlangen, so müssen die weissen Valenzen derjenigen Lichter, welche in verschiedenen Augen eine verschieden grosse Absorption erleiden mit der weissen Valenz eines Lichtes verglichen werden, das nicht absorbirt und also auch in seinem Reizwerth nicht verändert wird.

Ich habe die Absicht, eine solche Untersuchung, wenn sich mir die Gelegenheit bietet, auszuführen, wenngleich ich mir nicht verhehle, dass die Genauigkeit derselben beeinträchtigt wird durch die herabgesetzte Unterschiedsempfindlichkeit des für Dunkel adaptirten Auges, was hier um somehr in Betracht kommt, als in Hinblick auf die Fragestellung die zu vergleichenden Lichter unter einem das maculare Netzhautgebiet nicht iiberschreitenden Gesichtswinkel beobachtet werden müssten. 


\section{$\mathrm{Zu}$ T a fel VI.}

Die Curven, die ursprünglich in grösserem Maassstabe gezeichnet waren, erfuhren bei der Reduction nicht durehwegs genau dieselbe Verkleinerung; die oberste Reihe (die Curven I, IV, VII) ist am stärksten, die mittle Reibe (die Curven II, V, VIII) weniger, die untere Reihe (die Curven III, VI, IX) am wenigsten reducirt; die Ordinaten bei $D$ verhalten sich in den drei Reihen wie 40 : $42: 43$. Infolge dieser Ungleichmässigkeit der Reduction theile ich die Versuchsergebnisse auch noch in Zahlen mit.

\begin{tabular}{|c|c|c|c|c|c|c|c|c|c|}
\hline Wellenlänge & I. & $\mathbf{I I}$ & III & IV & V & VI & VII & VIIII & IX \\
\hline$\mu \mu 589$ (D) & 10,00 & 10,00 & 10,00 & 10,00 & 10,00 & 10,00 & 10,00 & 10,00 & 10,00 \\
\hline-560 & 9,66 & 9,56 & - & - & - & - & - & - & - \\
\hline 532,8 & - & $9, \mathbf{1 6}$ & - & - & - & 9,42 & - & - & - \\
\hline $526,9(\mathrm{E})$ & 9,36 & 8,90 & 9,80 & 9,00 & 9,59 & 9,17 & 9,30 & 9,16 & 9,13 \\
\hline $518,3\left(b_{1}\right)$ & 9,20 & 8,26 & - & - & 9,36 & 9,17 & 9,16 & 9,16 & 8,90 \\
\hline 515 & - & 7,90 & 9,23 & 一 & - & - & 8,73 & 8,66 & 8,83 \\
\hline 510 & 8,76 & 7,03 & - & - & - & - & - & 8,03 & 8,10 \\
\hline 505 & 7,76 & 6,56 & - & - & - & - & - & 7,71 & 7,43 \\
\hline 500 & 7,73 & 6,53 & 7,43 & $\dot{-}$ & 8,50 & 7,61 & 7,93 & 7,57 & - \\
\hline 495 & - & 6,10 & - & - & - & - & - & - & - \\
\hline $486,1(\mathrm{~F})$ & 6,86 & 6,60 & 7,06 & 6,56 & 7,85 & 7,39 & 7,73 & - & 6,66 \\
\hline 466,6 & - & 5,73 & -1 & - & - & 7,23 & - & - & - \\
\hline 438,4 & - & 5,73 & - & 6,40 & 7,60 & 6,85 & - & - & - \\
\hline $422,6(\mathrm{~g})$ & 6,50 & 5,10 & 6,56 & - & - & - & - & - & - \\
\hline
\end{tabular}

